\title{
OS DESAFIOS DA COMUNICAÇÃO NA REDUÇÃO DO RISCO DE INUNDAÇÃO
}

\author{
Murilo Noli da Fonseca \\ Pontifícia Universidade Católica do Paraná (PUCPR) \\ Programa de Pós-Graduação em Gestão Urbana, Curitiba, PR, Brasil \\ murilonoli@gmail.com \\ Carlos Mello Garcias \\ Pontifícia Universidade Católica do Paraná (PUCPR) \\ Programa de Pós-Graduação em Gestão Urbana, Curitiba, PR, Brasil \\ carlos.garcias@pucpr.br
}

\begin{abstract}
RESUMO
Reduzir o risco de inundação é um dos principais desafios que o poder público e as pessoas possuem na busca pela construção de cidades e sociedades resilientes. A comunicação emerge como um dos principais instrumentos de gestão para o alcance desse objetivo. Embora seja um tema emergente e pouco explorado até o momento, os trabalhos realizados sobre comunicação de risco têm apresentado resultados enormemente elucidativos no que diz respeito à relação da gestão e dos indivíduos com os riscos, colaborando e servindo como base para a concepção de estratégias e políticas centradas na redução de risco de desastres. Baseado nessa circunstância, o presente artigo tem como objetivo discutir a comunicação de risco, refletir acerca de seu processo e dos principais elementos que a compõem, ao mesmo tempo em que se busca identificar os principais desafios que emergem nesse processo. Para tanto, empregou-se a pesquisa bibliográfica na literatura internacional e nacional como técnica de pesquisa. Deste modo, as possibilidades e obstáculos discutidos a partir dos desafios levantados possibilitam aos atores envolvidos a repensar a forma como ocorre o processo de comunicação no Brasil e, com isso, desenvolver estratégias mais eficazes que atendam diferentes grupos populacionais em circunstâncias socioespaciais com características distintas.
\end{abstract}

Palavras-chave: Gestão pública. Prevenção. Mitigação. Preparação. Informação.

\section{COMMUNICATION CHALLENGES IN REDUCING FLOOD RISK}

\begin{abstract}
Reducing the risk of flooding is one of the main challenges that the government and people have in the search for the construction of resilient cities and societies. Communication emerges as one of the main management tools for achieving this goal. Although it is an emerging and little explored topic to date, the work carried out on risk communication has presented enormously enlightening results regarding the relationship of management and individuals with risks, collaborating and serving as a basis for the design of strategies and policies focused on disaster risk reduction. Based on this circumstance, this article aims to discuss risk communication, reflect on its process and the main elements that compose it, while seeking to identify the main challenges that emerge in this process. For this, bibliographic research was used in international and national literature as a research technique. Thus, the possibilities and obstacles discussed from the challenges raised enable the actors involved to rethink the way the communication process occurs in Brazil and, thus, develop more effective strategies that meet different population groups in socio-spatial circumstances with different characteristics.
\end{abstract}

Keywords: Public management. Prevention. Mitigation. Preparation. Information.

\section{INTRODUÇÃO}

A frequência e a magnitude das inundações extremas cresceram recentemente (BERGHUIJS et al., 2017) e podem aumentar devido as mudanças climáticas e ao crescimento econômico e populacional ao longo dos rios (WINSEMIUS et al., 2016), da costa (VITOUSEK et al., 2017) e em áreas urbanas (KUNDZEWICZ et al., 2014). Diante dessa circunstância, torna-se cada vez mais importante que uma gestão de risco busque planejar e implementar estratégias de comunicação de risco de inundação aos atores locais, especialmente os mais vulneráveis (IRGC, 2017), determinar a melhor forma de fornecer essas informações, e motivar aqueles em situação de risco a reduzir o risco de inundação, desenvolvendo ações de prevenção, mitigação e preparação, de modo a torná-los cada vez mais resilientes. 
Embora seja um tema pouco explorado e a sua importância seja cada vez mais reconhecida no âmbito acadêmico, foi apenas recentemente que estudos almejaram preencher essa lacuna, procurando analisar o efeito de diferentes estratégias de comunicação sobre a percepção e as intenções de se preparar para a ocorrência de uma inundação (BOTZEN, DE BOER e TERPSTRA, 2013; FELDMAN et al., 2016). Eles mostram que a informação adaptada às necessidades de um indivíduo tem influência direta na percepção de risco, e que a participação ativa das pessoas - os receptores - pode ter um impacto positivo na resiliência, pois contribui para o desenvolvimento de capacidades sociais e para a redução do risco.

No Brasil, entretanto, a comunicação de risco ainda não é legitimada como um instrumento de redução de risco de desastre e encontra-se numa fase muito precária, devido, principalmente, às limitações dos órgãos de proteção e defesa civil (SORIANO e HOFFMANN, 2015). Três problemas emergem nesse caso: a sua marginalidade ou inexistência em termos de planejamento urbano (DOURNEL, GRALEPOIS e DOUVINET, 2015), nem todos os municípios possuem uma estrutura capaz de lidar com as inundações e a sua comunicação não se caracteriza efetivamente como tal, mas sim em uma transferência unidirecional de informações dos agentes locais para os atores locais (SORIANO e HOFFMANN, 2015).

Baseado nessa conjuntura, constitui-se objetivo deste artigo apresentar, entender e discutir o processo de comunicação sobre a redução do risco de inundação, destacando a sua importância e como ela pode ser planejada e executada para atingir seus objetivos principais. Propõe-se ainda debater os diferentes elementos que a compõem e buscar uma compreensão sobre os principais desafios que levam aos atores envolvidos a receber, compreender e transformar as informações de gestão de risco de inundação em medidas de prevenção, mitigação e preparação. Para tanto, é realizada uma pesquisa bibliográfica na literatura internacional e nacional a partir das principais bases de artigos disponíveis atualmente.

Portanto, o artigo encontra-se estruturado da seguinte forma: inicialmente é realizada uma fundamentação sobre comunicação de risco de inundação. Em seguida, o método aborda a técnica de pesquisa adotada durante a pesquisa. Os resultados obtidos são apresentados após, com a apresentação dos principais desafios que a comunicação enfrenta na redução do risco. Por fim, são feitas as conclusões do artigo, apresentando suas limitações e possibilidades de pesquisa futura.

\section{COMUNICAÇÃO DE RISCO}

A literatura sobre o tema tem sido substancial a partir da década de 1970, nomeadamente em relação as áreas da saúde, riscos tecnológicos e químicos, enquanto aquelas dedicadas aos perigos naturais são menos desenvolvidas (HÖPPNER, BRÜNDL e BUCHECKER, 2012). Aplicada ao risco, a comunicação ocupa uma posição central na sua gestão, interligando e facilitando fluxos de informações e diálogos entre os outros elementos da sua estrutura (IRGC, 2017) (Figura 1). Por essa razão, ela é vista como um instrumento necessário desde a identificação e avaliação da situação de risco até a implementação e monitoramento de medidas, perpassando pelas cinco fases do ciclo do desastre: prevenção, mitigação, preparação, resposta e recuperação. Desse modo, pode-se afirmar que sem o processo comunicacional, a compreensão e a redução de risco de desastres não podem ser praticadas.

Figura 1 - Posição da comunicação na gestão de risco.

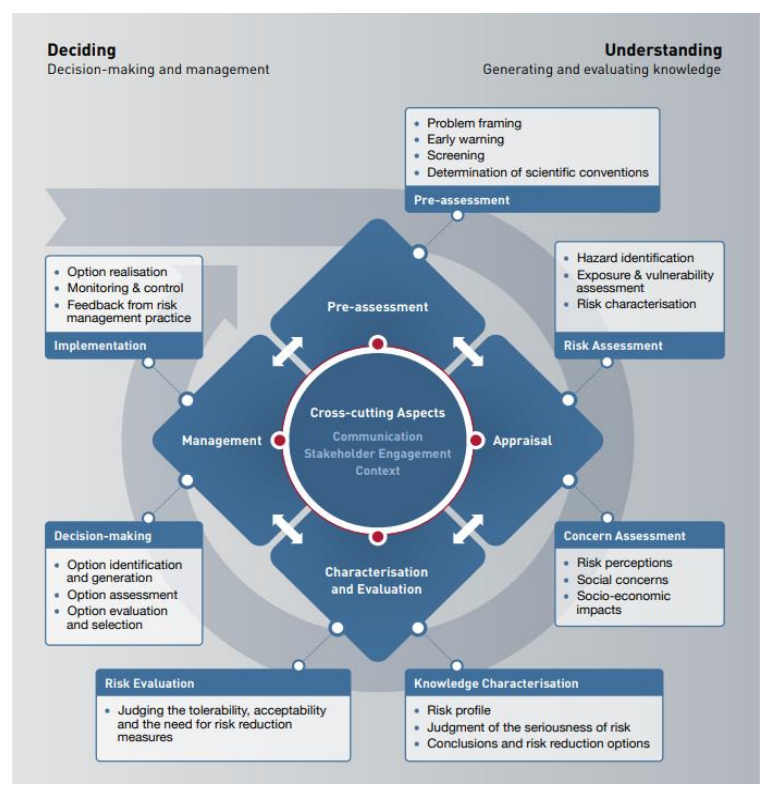

Fonte - IRGC (2017). 
Além de ser um instrumento de gestão de risco, a comunicação faz parte do direito das pessoas de serem informadas dos riscos. A Convenção de Aarhus (1981) estabelece os direitos dos cidadãos à informação, a serem ouvidos e a ter esses interesses adequadamente considerados. Dessa maneira, a comunicação de risco pode ser entendida como um processo social de troca de informações e avaliações claras e explicativas sobre o nível e o significado de qualquer forma de risco (HÖPPNER, BRÜNDL e BUCHECKER, 2012), bem como decisões, ações ou políticas para gerenciá-los (WOODS et al., 2012) entre diversas entidades - públicas e privadas - em todos os estágios de risco e em múltiplas dimensões espaciais (DEMERITT e NORBERT, 2014). Ela pode ocorrer de forma intrainstitucional, interinstitucional, midiático e comunitário. E contempla informações a respeito das peculiaridades intrínsecas ao risco, como a sua natureza e dimensão espaço-temporal, e à gestão, como as medidas estruturais e não-estruturais adotadas para a redução do risco, possibilitando assim uma melhor compreensão pelos atores locais.

Ela também pode ser vista como uma transferência unidirecional de informações relacionadas a perigos e riscos e sua gestão, e como troca bidirecional de informações, conhecimentos, atitudes e/ou valores relacionados (HAGEMEIER-KLOSE, 2008). Ela é compreendida como sendo uma atividade preventiva que pode melhorar o conhecimento sobre o risco (como agir), persuadir os cidadãos a mudar suas atitudes e/ou comportamentos, promover confiança nas autoridades e proporcionar as condições para um envolvimento efetivo das partes interessadas (RENN, 2008). Em outras palavras, prepara os atores envolvidos para a ocorrência do fenômeno, permitindo-lhes lidar melhor com o evento e a reduzir os impactos, danos e prejuízos decorrentes dele sobre os sistemas urbano e social, tornando-os resilientes.

Vale destacar que a comunicação de risco não é sinônimo de comunicação de desastres, de crises e de emergências. Esses últimos processos de comunicação são reativos e se concentram em atividades que envolvem um evento que já ocorreu e produziu danos (BEAN et al., 2015).

\section{COMUNICAÇÃO DE RISCO DE INUNDAÇÃO}

Aplicada ao risco de inundação, a comunicação almeja identificar áreas em risco de inundação, alcançar a atenção do maior número de pessoas nessa situação, divulgar informações sobre o risco, aumentar e melhorar a compreensão do fenômeno ao possibilitar que saibam a sua relevância e quando é provável a sua ocorrência, e, com isso, auxiliar na tomada de decisão (MAIDL e BUCHEKER, 2015). Com o objetivo de informar, educar e preparar as partes interessadas, por exemplo, podem ser organizados campanhas de conscientização e disseminação de informações sobre essa tipologia de risco; programas acadêmicos e de informação, educação e preparação; exercícios de defesa e evacuação; levantamento das consequências das inundações e de marcas históricas; compilação e disseminação de mapas de risco; formação de agentes locais; desenvolvimento de programas acadêmicos e de pesquisa sobre o tema; e estratégias de precaução e enfrentamento (THIEKEN et al., 2016; ZAHARIA e IOANA-TOROIMAC, 2016).

Portanto, a comunicação de risco de inundação deve auxiliar as pessoas a 1) saber se são vulneráveis; 2) compreender até que ponto estão sujeitos a esse fenômeno; e 3) permitir que se preparem melhor para uma inundação. Algumas perguntas são necessárias neste momento: 1) até que ponto a água pode subir, onde e quando? 2) quem é afetado, por que e como? 3) que tipo de informação e recomendação as pessoas precisam para responder efetivamente à inundação? 4) como fornecer informações relevantes para a população em risco? Para tanto, deve-se diagnosticar e analisar a situação de risco, identificar stakeholders capazes de gerenciar e comunicar o risco, escolher o objetivo da comunicação, escolher uma estratégia de comunicação, preparar mensagens informativas acessíveis e compreensíveis a todos, organizar um diálogo, compartilhar opiniões com o público e avaliar as medidas tomadas.

Contudo, não há uma relação direta entre transmitir informações sobre riscos e pessoas ou grupos populacionais que tomam decisões para reduzi-los. Receber, interpretar e entender a mensagem transmitida depende das características do receptor e de sua consciência de risco, bem como do contexto sociocultural (ROLLASON et al., 2018). Por essa razão, diversas recomendações são feitas para que o processo seja eficaz. Dentre elas, (1) conhecer o público-alvo; (2) não usar jargão, acrônimos ou eufemismos; (3) manter o vocabulário o mais familiar possível; (4) usar termos técnicos quando necessário e defini-los tanto no início como no contexto; (5) desenvolver um fluxo simples e fácil de ideias relacionadas; (6) produzir material em partes lógicas e compreensíveis; (7) desenvolver adequadamente cada conceito; e (8) resumir e repetir materiais para entendimento (CHESS e HANCE, 1994).

Assim, a comunicação de risco pode ser vista como um aspecto-chave para a capacitação social, tanto em termos de aumentar a competência e motivação das pessoas para agir quanto em termos de dar as condições necessárias para uma participação eficaz em nível comunitário (HOPPNER, BRÜNDL e 
BUCHECKER, 2012). Ou seja, os indivíduos com baixa consciência e interesse passam a ter a chance de se envolver no processo de definição de suas necessidades de informação (KJELLGREN, 2013).

\section{METODOLOGIA}

A consecução do objetivo proposto no presente trabalho foi realizada a partir de uma pesquisa bibliográfica. Trata-se de um procedimento que possibilita analisar os principais trabalhos já realizados, especialmente aqueles importantes na área e capazes de fornecer dados atuais e relevantes sobre o tema (LAKATOS e MARCONI, 2003). Ou seja, "oferece meios para definir, resolver, não somente problemas já conhecidos, como também explorar novas áreas onde os problemas não se cristalizaram suficientemente" (MANZO, 1971, p.32). Dessa maneira, foram analisadas publicações internacionais e nacionais contidas nas principais bases de dados que existem atualmente, sendo elas: Web of Science, Scopus, Portal de Periódicos CAPES, Scientific Electronic Library Online (SciELO) e Google Scholar. Para tanto, mapeou-se os estudos utilizando-se as seguintes palavras-chave: "comunicação" e "risco de inundação", as quais foram consideradas em cinco idiomas diferentes (português, espanhol, inglês, alemão e francês).

\section{OS PRINCIPAIS DESAFIOS}

\section{PARTICIPAÇÃO PÚBLICA}

A forma como comunicar o risco variou ao longo do tempo, sobretudo no que diz respeito à incorporação do público nesse processo. Passa-se de modelos simples de comunicação para abordagens mais complexas que levam em conta o caráter multifacetado dos riscos e da sociedade. Inicialmente, é concebido de uma maneira unidirecional entre comunicadores (gestores, especialistas) e destinatários (cidadãos), visando transmitir informações baseadas em probabilidades, conhecimentos e atitudes que os especialistas consideram mais importante (FELDMAN et al., 2016). Ele é realizado de maneira vertical por meio de mídias impressas e digitais, onde os indivíduos podem ou não ter dificuldades em ler ou receber.

No entanto, esse modelo tem sido criticado e classificado como produtor de um "déficit de informação" e de vulnerabilidade social (DEMERITT e NOBERT 2014), ao marginalizar as redes locais de informação e comunicação. A informação não é vista como confiável ou digna de ser mantida como tal pelo público ou são inconsistentes de várias fontes, e os que estão em risco são frequentemente enquadrados como necessitados e dependentes de especialistas para ditar quais informações de risco são importantes e por quê (BUBECK, BOTZEN e AERTS, 2012). Ou seja, os indivíduos normalmente são excluídos dos processos de criação de comunicações de risco. Logo, a dependência das autoridades pode enfraquecer a motivação para agir, inibir as práticas e dinâmicas desenvolvidas pelas comunidades, mas pode tornar mais provável que o grupo-alvo leve as informações fornecidas a sério (MAIDL e BUCHECKER, 2015).

De uma forma geral, as campanhas de comunicação que usam a abordagem vertical e entregam mensagens padronizadas em áreas de extenso território e cujas populações apresentam características distintas, tem dificuldades em promover uma maior aceitação dessas informações. Na Inglaterra, Países Baixos e Flandres, as campanhas governamentais unidirecionais não foram bem sucedidas em motivar as pessoas a tomar medidas de proteção (INTERREG, 2013), uma vez que não abordaram as diferentes atitudes que as pessoas têm em relação às inundações, especialmente em virtude da existência de diferenças socioeconômicas, culturais e individuais. Com isso, essa forma de comunicação pode ser restritiva e problemática, sobretudo por considerar o comportamento humano como consequência de um déficit de informação. Além disso, uma comunicação de risco de inundação sem a possibilidade de feedback sobre o seu êxito possui uma chance menor de sucesso do que uma estratégia bidirecional.

Entretanto, essa forma de comunicar o risco ainda permanece como a forma mais empregada na gestão pública. Trata-se de uma circunstância que ocorre especialmente em virtude da (1) baixa avaliação do interesse do público na comunicação; (2) medo dos gestores públicos de desencadear uma reação negativa (3) falta percebida de recursos para se comunicar com sucesso; (4) e falta de pressão por parte do Estado, juntamente com falta de apoio e exemplos de boas práticas (KJELLGREN, 2013). Como forma de ter maior êxito no processo de conscientização, foi reconhecido que o comportamento individual tende a refletir uma infinidade de fatores atrelados a aspectos sociais e psicológicos. Pois as pessoas não são apenas passivas, elas também são destinatárias ativas de informações que são avaliadas individualmente.

É importante ressaltar que não existe um público homogêneo. Diferentes grupos populacionais com distintos perfis sociais e demográficos podem necessitar de distintas estratégias de comunicação (HÖPPNER, BRÜNDL e BUCHECKER, 2012). Por isso, a comunicação centrada nas necessidades

$\begin{array}{lllll}\text { Caminhos de Geografia } \quad \text { Uberlândia-MG } & \text { v. 22, n. } 81 & \text { jun./2021 } & \text { p. 01-14 } & \text { Página } 4\end{array}$


especificas das partes interessadas, a partir dos atos de informar, consultar, envolver, colaborar e capacitar, é mais eficaz do que a abordagem tradicional vertical e o principal motivador de um indivíduo investir na redução do risco (HAER, BOTZEM e AERTS, 2016) (Figura 2). O conhecimento local é essencial para fortalecer a compreensão e a conscientização das comunidades e para comunicar o risco.

Figura 2 - Processo de comunicação bidirecional.

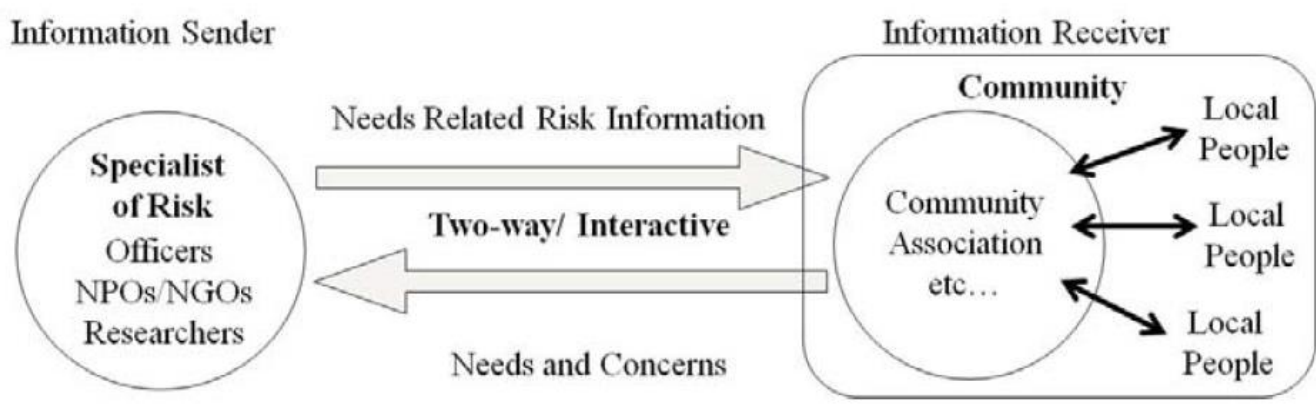

Fonte - Takeuchi (2006).

Ações dessa natureza podem ser potencializadas pelo desenvolvimento das tecnologias da informação e comunicação (TICs), as quais permitem que as comunidades locais forneçam informações e feedback instantâneo sobre as condições do ambiente em que residem.

\section{PERCEPÇÃO (ÕES) DE RISCO (S)}

A comunicação e a percepção de risco estão intimamente conectadas. Para comunicar um risco, ele deve primeiro ser percebido. Ou seja, no início do processo de comunicação, pelo menos o comunicador deve estar ciente do risco e de sua relevância, caso contrário, não veria necessidade de comunicá-lo. Em seguida, os destinatários também devem perceber o risco comunicado como tal e interpretá-lo como algo que Ihes diz respeito. Logo, a percepção tem influência no êxito da comunicação de risco, já que pode levar a diversos problema se ignorada, mas também é esperado que esse processo modele a percepção (PING et al., 2016). Geralmente, níveis mais altos de risco percebido aumentam a motivação de proteção, enquanto níveis mais baixos podem levar à negligência (NEUWIRTH, DUNWOODY e GRIFFIN, 2000).

Portanto, a percepção de risco é um processo pelo qual os riscos são subjetivamente, ou intuitivamente, compreendidos e avaliados por um indivíduo, grupo ou comunidade. É com base na vivência diferenciada do espaço que o ser humano desenvolve sua realidade social. Ou seja, representa o modo como os indivíduos organizam mentalmente o ambiente que o rodeia no âmbito individual (acesso a informações e a maneira como são divulgados, confiança, emoções, memórias, experiências, interesses, sentimentos, relações com o lugar, situação e posição) e cultural e social em que está inserido (religião, grupo étnico, família, situação econômica) (RENN, 2008; THIEKEN et al., 2016). Logo, as estratégias de comunicação de risco de inundação necessitam levar em conta essa diversidade, para que tenha um impacto positivo sobre as ações de prevenção, mitigação e preparação da comunidade a eventos extremos.

Um dos elementos que influencia no processo perceptivo é a memória. Ela possibilita recordar os eventos que acontecem com regularidade, aqueles mais significativos ou os mais recentes. Os eventos que ocorrem com maior regularidade e que afetam diretamente a vida das pessoas são facilmente lembrados e, por essa razão, têm sua probabilidade mais bem avaliada, em comparação àqueles pontuais e sem danos de grande monta às pessoas (KATES, 1978). Por conseguinte, o impacto da experiência passada nos indivíduos dura mais tempo se as consequências de um evento grave estiverem ancoradas na mente dos indivíduos (TERPSTRA, 2011). Entretanto, o risco pode estar presente em mente e, simultaneamente, as pessoas o negam veementemente para que possam planejar o futuro. Por isso mesmo, não acreditam que tais eventos ocorram de novo em um curto período (KATES, 1978).

Além dos elementos relacionados ao indivíduo, a percepção de risco é influenciada por elementos da própria situação de risco, como magnitude (força do evento), frequência (tempo médio de regresso de eventos extremos), duração (tempo de manifestação do fenômeno), área de alcance (extensão da área atingida), velocidade de deflagração (rapidez com que o evento ocorre), dispersão espacial (padrão espacial da ocorrência) e distribuição temporal (padrão temporal da distribuição) (SOUZA e ZANELLA, 
2010). Não se pode esquecer também dos próprios impactos (diretos e indiretos), danos e prejuízos decorrentes da ocorrência do evento como fatores capazes de influenciar a percepção. Assim, compreender o contexto em que a percepção ocorre é fundamental para a avaliação de riscos.

No entanto, diversos desafios surgem ao implementar uma estratégia de comunicação. O primeiro deles é chamar a atenção de pessoas que ainda não foram afetadas ou que apresentam uma baixa percepção e familiarizá-los com o tema e entregar informações que sejam compreensíveis. A "preocupação" foi identificada como um fator para explicar se as pessoas se informam sobre os riscos e se motivam a se preparar para um evento (RAAIJMAKERS, KRYWKOW e VAN DER VEEN, 2008). Criar consciência sobre o risco de inundação é uma tarefa de difícil execução, uma vez que as pessoas não estão particularmente interessadas nelas, especialmente em virtude da ausência do fenômeno e de seus efeitos no cotidiano (KJELLGREN, 2013). Dessa maneira, a percepção varia entre aqueles que desconhecem as ameaças (VESTENA et al., 2014), os que aguardam a ação do poder público na redução do risco (JACOBI, 2006), e aqueles que se submetem a eles por não ter outra local de moradia.

Além da consciência, a identificação dos elementos deflagradores de inundações é fundamental no estabelecimento de uma estratégia de comunicação que busca gerar ou aumentar a percepção de risco, tendo em vista que essas interpretações visam objetivos diferentes, geram contextos e emoções distintas ou justificam ações e medidas. Habitualmente, a responsabilidade pela ocorrência de inundações e pela situação de risco é imputada a elementos naturais e abstratos, e o papel do ser humano nesse processo é marginalizado (WHYTE, 1985). Nesse sentido, uma parcela de pessoas procura não se envolver em atividades relacionadas às condições ambientais do lugar em que reside (SOUZA e ZANELLA, 2010).

Deve-se compreender também o papel do poder público nesse processo. Xavier (1996) mostra que há uma aproximação entre a percepção dos indivíduos que residem em áreas de risco e a dos gestores públicos sobre o risco e as medidas a serem adotadas. Já Kammerbauer e Minnery (2018) afirmam que ambos percebem os riscos de maneiras díspares e tendem a utilizar termos diferentes para discuti-los. Pesquisas demonstram que a terminologia e a frase usadas para comunicar o risco de inundação pode afetar a forma como a informação é percebida (STRATHIE et al, 2017). Por exemplo, a informação numérica é mais bem compreendida quando apresentada no formato de frequência (por exemplo, $1 \mathrm{em}$ 20) do que como percentagem (por exemplo, $5 \%$ de chance) (GIGERENZER e HOFFRAGE, 1995). Por outro lado, os formatos que apresentam pequenas probabilidades e cumulativas recebem maior atenção quando carregados de afeto e imagens emocionais (ROTTENSTREICH e HSEE, 2001).

É imperioso lembrar que a percepção de risco varia conforme a experiência de cada indivíduo no espaço, por isso não existem "pessoas sem" e "pessoas com" percepção de risco. Os indivíduos que residem em regiões atingidas tendem a apresentar maior ciência do risco, estar mais bem preparadas e ter uma ideia melhor do que fazer quando há um alerta do que aqueles que vivem em regiões raramente afetadas (KIENZLER et al., 2015). Além do mais, percepções de risco elevadas resultam, normalmente, em maior busca de informações, principalmente quando se recebe diversas mensagens de informações de risco e conhece pessoas que adotam medidas preventivas. Entretanto, na Áustria, o governo tem feito com que os cidadãos se percebessem como de menor risco, tornando-os mais propensos a confiar na ação pública contra inundações (SEEBAUER e BABCICKY, 2017).

Baseado nisso, quatro padrões de conduta em face ao risco podem ser identificados:

1. O risco não é percebido: os indivíduos não têm consciência da ameaça, pois não acreditam na sua ocorrência. Comumente, tal conduta ocorre quando ainda não houve fortes impactos ou, se existiram, foram ínfimos e absorvidos facilmente.

2. O risco é percebido, mas aceito de forma passiva: as ameaças são reconhecidas e consentidas, pois são avaliadas como uma condição pela moradia.

3. O risco é minimizado perante ações de redução: os sujeitos possuem consciência do risco e medidas são postas visando reduzir a vulnerabilidade e, assim os impactos.

4. O risco provoca a alteração/transformação do uso do solo: o limite da intolerância foi atingido e o risco determina a transformação do uso do solo, a modificação do local de residência ou a combinação de ambos (SOUZA e ZANELLA, 2010).

Ao explorar essas questões que influenciam direta e indiretamente na percepção de risco de inundação, é possível otimizar estratégias de comunicação para garantir níveis apropriados de conscientização e o desenvolvimento de ações de prevenção, mitigação e preparação, tendo em vista que influencia na vulnerabilidade e na adaptação. Todavia, ainda são necessários esforços para entender as interações 
entre a percepção e a comunicação de risco, manter os níveis de informação e aceitação, aumentar o nível de preparação, envolver a população na gestão, bem como os processos sociais para alcançar percepções compartilhadas e medidas apropriadas.

\section{ELEMENTOS DA COMUNICAÇÃO DE RISCO DE INUNDAÇÃO}

\section{ATORES - COMUNICADOR E DESTINATÁRIO}

A comunicação de risco é realizada por uma vasta gama de atores cujas perspectivas devem ser consideradas em diversos níveis espaciais. Eles são nós em cadeias ou redes de comunicação entre as quais a informação flui em uma ou várias direções. A força, estabilidade, frequência e a direção do fluxo de informação e a centralidade dos atores são definidos a partir das características de tais redes (HÖPPNER, BRÜNDL e BUCHECKER, 2012). Dentre os atores que compõem esse processo, destacamse os cidadãos, instituições públicas e privadas, governo, sistema educacional, cientistas, organizações não-governamentais (ONG) e meios de comunicação de massa, como televisão, rádio e jornal.

Entretanto, faz-se necessário destacar quem são os atores responsáveis por cada etapa da comunicação, desde a construção do conhecimento científico do tema até o período pós ocorrência do fenômeno. Até mesmo porque, em alguns casos pode haver algum nível de confusão sobre quem está fazendo o quê, onde e quando (PING et al., 2016). Primeiramente há aqueles que estão na fonte de informações sobre as inundações. São os especialistas, cujo trabalho envolve o desenvolvimento da base científica que sustenta a gestão de riscos. Esse grupo inclui geógrafos, engenheiros, hidrólogos, meteorologistas e economistas, que desenvolvem modelos de falha de infraestruturas críticas (FAULKNER, MCCARTHY e TUNSTALL, 2010), como aquelas atreladas ao saneamento, saúde, energia, comunicação e transporte.

Segundo, há os profissionais cujo trabalho envolve as ações de gestão de risco de inundação (FAULKNER e BALL, 2007). São eles planejadores, gestores e agentes de seguros. Terceiro, o público, os receptores da comunicação. Mas, o termo "público" não se limita aos habitantes de áreas de risco. Ele abrange outros atores, como o setor privado, setor de seguros, gestores e jornalistas. Tais profissionais têm responsabilidades não apenas de se comunicar na interface pública, mas também de atuar como uma fonte de informação entre si e com outras instituições (FAULKNER, MCCARTHY e TUNSTALL, 2010).

\section{CONTEÚDO DA MENSAGEM}

Desenvolver e apresentar uma mensagem é essencial para tornar a comunicação de risco eficaz. O modo como uma mensagem é desenvolvida influencia na forma como as pessoas interpretam e respondem à emissão de avisos e alertas, bem como a origem da informação, a consistência e precisão nas mensagens, a credibilidade percebida da fonte (MILETI e SORENSEN, 1990). Elas permitem que as pessoas tenham conhecimento dos riscos a que estão expostas e adotem comportamentos e atitudes apropriados antes, durante e depois da ocorrência de uma inundação. Por essa razão, a mensagem inicial é importante para ganhar o interesse das pessoas. No entanto, uma mensagem que visa informar as pessoas sobre as medidas que devem ser tomadas para a sua proteção será inútil se o seu conteúdo for restrito a textos e não for adaptado às características dos destinatários, como estilo de aprendizagem, gênero, alfabetização, experiência com o fenômeno, ou se não houver uma confiança no emissor. Logo, levará a busca de mais informações, atrasando a adoção de medidas de proteção (WOODS et al., 2012). Por outro lado, o uso de meios visuais e convencionais de apresentação (ilustrações, gráficos, mapas, linhas de tempo) geralmente é melhor do que apenas texto (LUNDGREN e MCMAKIN, 2013).

Desse modo, o conteúdo da mensagem varia conforme a fase da gestão de risco. No cotidiano, quando existe o risco de inundação, a comunicação será empregada para transmitir informações gerais sobre onde, o que e como as informações sobre riscos de inundação podem ser encontradas, lidas e interpretadas. Com o objetivo de fortalecer uma conscientização, os atores locais devem ser informados periodicamente sobre os perigos, os níveis de risco a que estão expostos, o que ocorreu nos eventos passados (memória), e medidas estruturais e não-estruturais, nomeadamente sobre planejamento, capacitação e fortalecimento da confiança. Logo, o desafio é garantir que as informações fornecidas sejam expressivas para os destinatários e os motivem a adotar medidas de proteção e redução do risco

$\mathrm{Na}$ iminência do evento, tanto as pessoas em risco quanto aqueles que não estão nessa situação precisam ser alertadas sobre a ocorrência do evento. Cada mensagem de alerta deve incluir a natureza, a localização, a orientação, o tempo e a fonte do evento ou de risco esperado (SORENSEN, 2000). Os alertas podem ser emitidos pelo governo local, por vizinhos, parentes ou resultantes do compartilhamento 
de informações via mídias sociais. Na Nova Zelândia, por exemplo, a maior parte dos entrevistados indicaram que as informações mais importantes nesse momento são: localização e tempo necessário para chegar a um abrigo, rotas de evacuação e proteção contra roubos e saques (GUTTELING et al., 2010).

Durante um alerta, a comunicação é sobre previsão, medidas de proteção, regras comportamentais, evacuação e localização de abrigos públicos e/ou locais seguros próximos. Ou seja, mensagens eficazes elucidam quem deve e quem não deve ser proteger e quando iniciar e concluir essas atividades em termos que o público possa entender (PARKER, PRIEST e MCCARTHY, 2011). Também, devem responder às seguintes perguntas: qual é o perigo? O que os indivíduos devem fazer? Quanto tempo resta antes do impacto? As informações são comunicadas com frequência em vários canais? As fontes são oficiais? A informação pode ser confirmada? (MILETI e O'BRIEN, 1992). Normalmente, o governo local decide se um alerta precisa ou não ser emitido. Se o processo de comunicação anterior a este momento foi realizado de maneira eficaz, é mais provável que as pessoas se protejam durante o evento, pois apresentam consciência e sabem quais medidas devem ser adotadas (LAMOND et al., 2019).

Já quando há o recebimento dos alertas, a percepção é moldada por "ouvir - confirmar - entender acreditar - personalizar - responder" (MILETI e O'BRIEN, 1992, p. 41). A informação que as pessoas recebem está sujeita a interpretações. Em outras palavras, mesmo que todos estejam ouvindo a mesma mensagem, diferentes indivíduos podem chegar a conclusões distintas sobre o que ouvem (MILETI e SORENSEN, 1990), inclusive em relação àqueles que originalmente a produziram. Por essa razão, um dos principais fatores que influencia na eficácia da resposta é o descompasso entre o conteúdo da mensagem e as características das pessoas e do ambiente em que residem (Figura 3).

Figura 3 - Variáveis que determinam a resposta ao alerta de inundação.

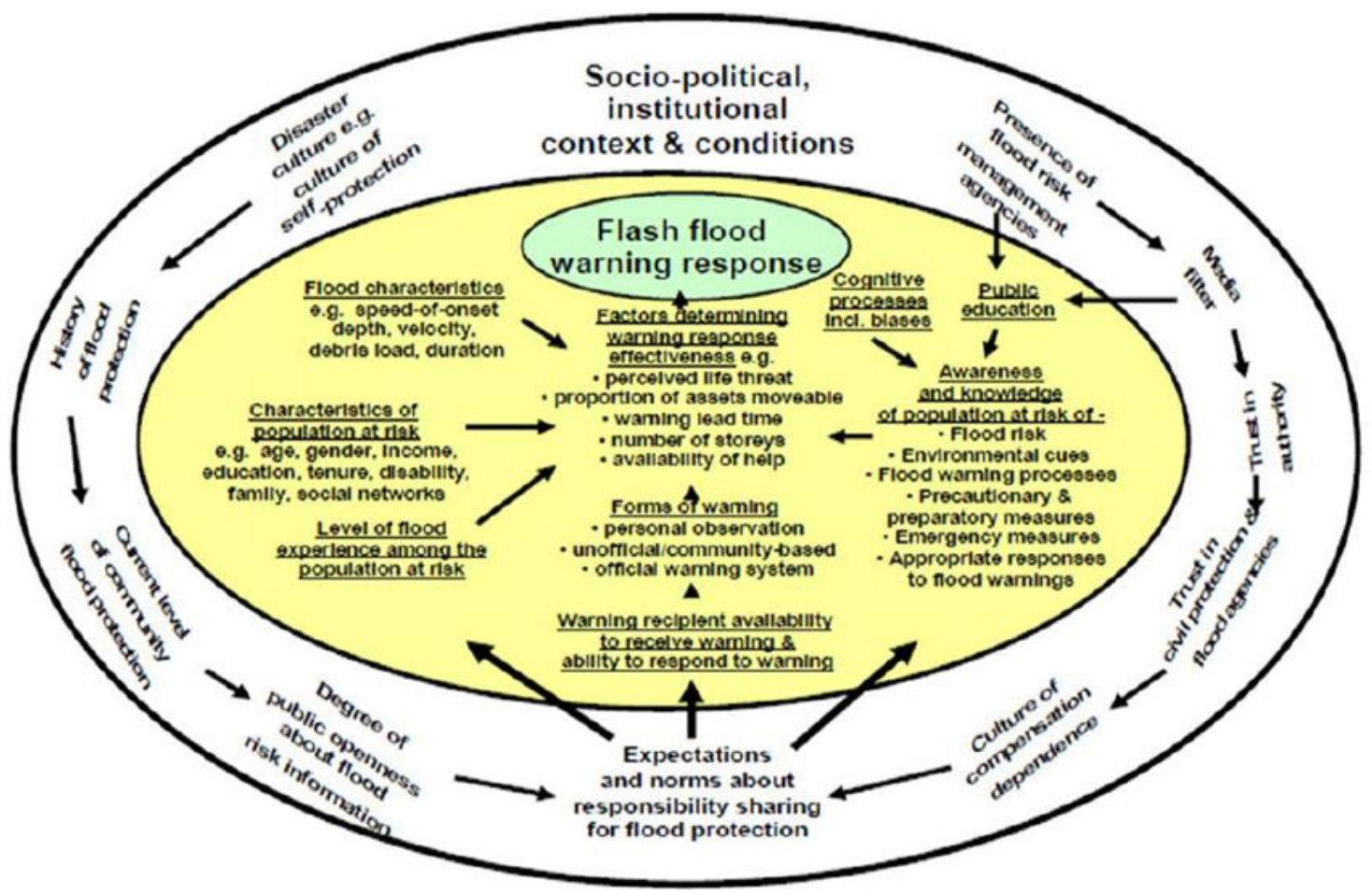

Fonte - Parker - Tunstall e Mccarthy (2007).

Em Roterdam, Holanda, e Wealdstone, Inglaterra, por exemplo, os indivíduos com experiência recente afirmam que perderam confiança nos alertas após repetidas falhas na sua emissão, especialmente os eventos que ocorreram sem o alerta (PARKER, PRIEST e MCCARTHY, 2011). Os participantes da cidade inglesa também relataram o recebimento de alertas vagos e inespecíficos emitidos por uma agência e contraditos por outra. Já em Brisbane, Austrália, durante as inundações ocorridas em 2011, a comunicação foi comprometida por uma desconexão entre a natureza especializada e técnica da informação e como os residentes a interpretavam ao tomar suas decisões (KAMMERBAUER e 
MINNERY, 2018). Também, a comunidade não havia sido capacitada para compreender a extensão do risco de inundação, interpretar alertas, decodificar mapas e formular estratégias de evacuação.

Como forma de minimizar isso, estratégias devem ser adotadas. Por exemplo, a observação participante, entrevistas com líderes comunitários, grupos focais com especialistas e pessoas residentes em áreas de risco, discussões sobre o pós-evento. Isto é, formas de compreender se o alerta levou ou não à ocorrência e quais foram os obstáculos e fatores de sucesso, a fim de aprimorá-lo. Um sistema de monitoramento e alerta precisa ser complementado por campanhas de conscientização e treinamento, sobretudo em áreas raramente afetadas, e ser coproduzido pelos atores locais, empregando uma base de dados capaz de ser atualizada à medida que as inundações ocorrem (PARKER, PRIEST e MCCARTHY, 2011).

A ocorrência propriamente dito de uma inundação envolve a comunicação sobre a cota de inundação a ser atingida, medidas de evacuação, localização de abrigos ou locais seguros e regras comportamentais. Para apoiar esse processo de tomada de decisão, faz-se necessário complementar a mensagem de alerta com informações sobre potenciais impactos, uma vez que o comportamento humano durante a ocorrência de inundações é influenciado pelo seu conhecimento e experiência com o risco (QUARANTELLI, 2008). Ou seja, se a informação é divulgada logo após o evento, as mensagens se tornam insignificantes à luz da experiência vivida. Por outro lado, se a informação ocorre em áreas onde as últimas inundações são antigas, as mensagens colidem com formas de esquecimento ou negação (HAGEMEIER-KLOSE, 2008).

Após uma inundação, normalmente são comunicadas medidas de recuperação, como informações sobre trabalhos de limpeza e reconstrução, as causas e os antecedentes do evento. No entanto, também se trata de aprender com o evento (obstáculos e fatores de sucesso), avaliar a gestão de riscos e ajustá-lo. Essa fase retorna à vida cotidiana, em que a prevenção é o elemento central da comunicação de riscos.

\section{MODOS, CANAIS E FERRAMENTAS}

A comunicação de risco ocorre através de diferentes modos, canais e ferramentas, que devem ser convergentes e orientados pelos seus propósitos e funções. O modo pode ser escrito (jornal, carta e relatório, etc.), verbal (contação de histórias e rodas de conversa, etc.) e não verbal/visual (linguagem corporal e gestual, gráficos, filmes, etc.) (HÖPPNER, BRÜNDL e BUCHECKER, 2012). Já os canais são face-a-face ou mediada. Eles visam um público específico ou difuso, que pode ser um, poucos ou muitos atores. No que se refere ao aspecto temporal, pode ser uma atividade única ou contínua (HÖPPNER, BRÜNDL e BUCHECKER, 2012).

Diversas ferramentas para visualizar informações sobre riscos de inundação foram desenvolvidas em diversos países, as quais podem ser assistidas ou não por tecnologia. As ferramentas tradicionais incluem fontes impressas (jornais, revistas e folhetos, etc.); eletrônicas (televisão, rádio, telefone, filmes, documentários, internet, etc.); comunicações pessoais; aulas e atividades temáticas; visitas de estudo; eventos e ações públicas; e marcas na paisagem (HAGEMEIER-KLOSE, 2008). Por exemplo, uma comunicação unidirecional pode apresentar pouca ou nenhuma interatividade, como materiais impressos, ou dirigida para o diálogo, como a participação (HÖPPNER, BRÜNDL e BUCHECKER, 2012).

Além dessas ferramentas, as mídias sociais vêm crescendo como mecanismos de comunicação de informações sobre os riscos naturais (FELDMAN et al., 2016). Elas geralmente se referem a aplicativos baseados na Internet que facilitam o compartilhamento de informações em computadores e dispositivos móveis, como as redes sociais (por exemplo, Facebook e Twitter), mensagens de texto, salas de batepapo, fóruns de discussão e blogs (LINDSAY, 2011). O uso de mídias sociais também tem sido cada vez mais utilizados por agências governamentais, uma vez que é considerado como um veículo efetivo para o compartilhamento de informação para vários públicos, estabelecendo um veículo para uma comunicação bidirecional entre cidadãos e governo (DZIAŁEK et al., 2019).

Até mesmo devido a existência de múltiplos canais de comunicação, as fontes escolhidas pelos cidadãos para receber informações confiáveis são diversas. Para garantir o efeito desejado, uma variedade de fontes, tradicionais e eletrônicas, adaptadas a pessoas, grupos, comunidades e áreas, considerando o contexto sociocultural local, deve ser utilizada para comunicar o risco e emitir avisos e alertas de maneira eficaz (O'SULLIVAN et al., 2012). Por exemplo, as pessoas na maioria das vezes confiam em fontes consideradas concisas, credíveis, confiáveis, atenciosas, informadas, especializadas e honestas, cujas informações devem ser relevantes e capazes de prescrever alguma resposta (FELDMAN et al., 2016).

As fontes de informação mais utilizadas antes e durante uma inundação é o rádio e a televisão (LAMOND et al., 2019). Isto sugere que as fontes de informação baseadas na web não são uma escolha universal 
para receber informações de risco. Ainda, a idade é um dos elementos que prediz o uso ou a preferência por tipos de fontes de informação de risco (FELDMAN et al., 2016). Enquanto a internet é uma importante fonte de informação de risco sobretudo entre os mais jovens, os idosos tendem a utilizar canais tradicionais de comunicação como fonte de informação, como jornais, revistas, folhetos, TV, rádio, telefone e boca-a-boca (LINDEL e PERRY, 2012). Eles também não desejam receber informações através de mídias sociais (BEAN et al., 2015).

\section{CONCLUSÃO}

Discutir as concepções de comunicação de risco de inundação, compreender os principais elementos que a compõem, como as suas características, estratégias, limites e possibilidades de planejamento e execução, e sua relevância para a gestão pública, sobretudo a local, são passos necessários para repensar a forma como ocorre o processo de identificação, avaliação e divulgação de informações sobre o risco, a fim de reduzi-lo e construir sistemas e sociedades cada vez mais resilientes. Embora seja um tema emergente e pouco explorado, há um crescente interesse nesta área na comunidade científica, sinalizando uma busca pelo aprofundamento do conhecimento e da base conceitual sobre o tema.

A partir dos desafios levantados e discutidos, sugere-se que pesquisas futuras examinem, por exemplo, qual é o nível de eficácia das estratégias de comunicação de risco na antecipação e preparo de populações vulneráveis no enfrentamento de inundações. Como ponto de partida, é necessário identificar quem se comunica, ou seja, quem são os atores envolvidos no processo de gestão e comunicação de risco de inundação, bem como se ocorre a participação pública, e, em seguida, verificar a existência de (des) articulações nesse processo. Por exemplo, uma mesma atividade, como o mapeamento, pode ser realizada de forma individual e sem diálogo por dois órgãos públicos, o que levará a resultados distintos e, assim, a uma comunicação conflituosa. Ainda no âmbito institucional, deve-se entender como ocorre o fluxo de informações entre as áreas setoriais de uma gestão pública, bem como os canais e ferramentas utilizadas para tal. Ou seja, se as informações produzidas por um órgão subsidiam as ações de outro e se elas apresentam características de qualidade, permitindo uma tomada de decisão mais eficaz.

Posteriormente, é vital analisar como os processos descritos anteriormente impactam na comunicação do agente emissor (normalmente o poder público) para os demais atores sociais, sobretudo os vulneráveis. Com isso, deve-se avaliar o que é comunicado (conteúdo), quais são os objetivos da comunicação (criar confiança e mudar o comportamento, por exemplo), como (modos, canais, ferramentas) e quando isso ocorre (cotidiano - prevenção e mitigação, iminência do evento - preparação, ocorrência da inundação resposta, e pós-evento - recuperação), e quais são os pontos positivos e negativos decorrentes dele. Uma característica que requer maior atenção nesse âmbito, e especialmente na atualidade, é como os gestores públicos e demais agentes comunicadores podem gerenciar notícias falsas (fake news) sobre os riscos.

Em um segundo domínio, deve-se avaliar o contexto e processo o qual molda a forma como as pessoas e grupos sociais processam, interpretam e reagem às informações e alertas de inundação. Não obstante, algumas questões emergem perante esse processo: quais são as expectativas dos destinatários em relação aos riscos de inundação? existe alguma forma vigente da população conhecer os riscos? Ela tem acesso às informações de risco? A gestão local apresenta as ferramentas utilizadas para a comunicação? Como isso ocorre? Como essas ferramentas são avaliadas pelos receptores? Em que medida as expectativas da população são atendidas pelas ferramentas de informação utilizadas pela gestão pública? Por exemplo, até que ponto os gestores locais fazem reuniões com os grupos vulneráveis para disseminar e discutir informações sobre os riscos? Ou, qual é a melhor forma de isso ocorrer?

Também, as informações fornecidas pelo comunicador sobre os riscos de inundação são percebidas e compreendidas? Se sim, como? Se não, por que não? As informações de risco de inundação têm impacto no destinatário? Em caso afirmativo, eles têm o efeito pretendido do comunicador? Se não, por que não? Não menos importante, é avaliar até que ponto os cidadãos estão realmente interessados em receber informações e alertas de risco de inundação. Ou, a estrutura social individualista na sociedade líquida hodierna dificulta processo de comunicar o risco, inibindo ações que possibilitam uma melhor forma de lidar com a ocorrência do fenômeno e as consequências decorrentes deles?

\section{AGRADECIMENTOS}

O presente trabalho foi realizado com apoio da Coordenação de Aperfeiçoamento de Pessoal de Nível Superior - Brasil (CAPES) - Código de Financiamento 001. 


\section{REFERÊNCIAS}

BEAN, H., SUTTON, J., LIU, B. F., MADDEN, S., WOOD, M. M., MILETI, D. S. The Study of Mobile Public Warning Messages: A Research Review and Agenda. Review of Communication, vol.15, n.1, p.60-80, 2015. https://doi.org/10.1080/15358593.2015.1014402

BERGHUIJS, W.R., AALBERS, E.E., LARSEN, J.R., TRANCOSO, R., WOODS, R.A. Recent changes in extreme floods across multiple continents, Environmental Research Letters, vol.12, p.1-8, 2017. https://doi.org/10.1088/1748-9326/aa8847

BOTZEN, W.J.W., DE BOER, J., TERPSTRA, T. Framing of risk and preferences for annual and multiyear flood insurance. Journal of Economic Psychology, vol.39, p.357-375, 2013.

https://doi.org/10.1016/j.joep.2013.05.007

BUBECK, P, BOTZEN, W.J.W., AERTS, J.C.J.H. A review of risk perceptions and other factors that influence flood mitigation behavior: review of flood risk perceptions. Risk Analysis, n.32, p.14811495, 2012. https://doi.org/10.1111/j.1539-6924.2011.01783.x

CHESS, C., HANCE, B.J. Communicating with the Public: Ten Questions Environmental Managers Should Ask, Center for Environmental Communication, Rutgers University, New Brunswick, 1994

DEMERITT, D., NORBERT, S. Models of best practice in flood risk communication and management. Environmental Hazards, n.13, p.313-328, 2014. https://doi.org/10.1080/17477891.2014.924897

DOURNEL, S., GRALEPOIS, M., DOUVINET, J. Les projets urbains en zones inondables communiquent-ils sur les risques? Regard sur les politiques d'aménagement de quartiers à SaintÉtienne, Orléans et Nantes. Belgeo. Revue belge de géographie, vol.1, p.1-24, 2015. https://doi.org/10.4000/belgeo.16691

DZIAŁEK, J., BIERNACKI, W., KONIECZNY, R., FIEDEŃ, L., FRANCZAK, P., GRZESZNA, K., LISTWAN-FRANCZAK, K. Online Flood Risk Communication. In: DZIAŁEK, J., BIERNACKI, W., KONIECZNY, R., FIEDEŃ, L., FRANCZAK, P., GRZESZNA, K., LISTWAN-FRANCZAK, K. Understanding Flood Preparedness Flood Memories, Social Vulnerability and Risk Communication in Southern Poland. SpringerBriefs in Geography. 2019. https://doi.org/10.1007/978-3-030-04594-4

FAULKNER, H., BALL, D. Environmental hazards and risk communication. Environmental Hazards, vol.7, n.2, p.71-78, 2007. https://doi.org/10.1016/j.envhaz.2007.08.002

FAULKNER, H., MCCARTHY, S., TUNSTALL, S. Flood risk communication. p.386-406. 2010. In: G. Pender e H. Faulkner (Eds.), Flood risk science and management, Blackwell, 2010. https://doi.org/10.1002/9781444324846.ch19

FELDMAN, D., CONTRERAS, S., KARLIN, B., BASOLO, V., MATTHEW, R., SANDERS, B., LUKE, A. Communicating flood risk: looking back and forward at traditional and social media outlets.

International Journal of Disaster Risk Reduction, vol.15, p.43-55, 2016. https://doi.org/10.1016/j.ijdrr.2015.12.004

GIGERENZER, G., HOFFRAGE, U. How to improve Bayesian reasoning without instruction: Frequency formats, Psychological Review, vol. 102, n.4, p.684-704, 1995. https://doi.org/10.1037/0033-295X.102.4.684

GUTTELING, J.M., BAAN, M., KIEVIK, M., STONE, K. "Geen paniek! Risicocommunicatie door de ogen van de 'burger'. In: VAN DER MOST, H., DE WIT, S., BROEKHANS, B., ROSS, W. Kijk op Waterveiligheid, Delft: Eburon, 2010.

HAER, T., BOTZEN., W. J. e AERTS, J. C. J. H. Integrating household mitigation behaviour in food risk analysis: an agent-based model approach. Risk Analysis. p.1-15, 2016.

https://doi.org/10.1111/risa.12740 
HAGEMEIER-KLOSE, M. Hochwasserrisikokommunikation zwischen Wasserwirtschaftsverwaltung und Öffentlichkeit. Eine Evaluation der Wahrnehmung und Wirkung behördlicher Informationsinstrumente in Bayern. 196p. Tese (Doutorado em Filosofia). Universidade Técnica de Munique. 2008

HÖPPNER, C., BRÜNDL, M., BUCHECKER, M. Risk Communication and Natural Hazards. CapHaz project. Birmensdorf, Switzerland, 2012

INTERREG - EUROPEAN TERRITORIAL CO-OPERATION. Raising Flood Awareness and Self Efficacy - Framework to Develop and Implement a Successful Social Marketing Programme. 2013

IRGC - INTERNATIONAL RISK GOVERNANCE COUNCIL. Risk governance: coping with uncertainty in a complex world: Routledge. 2017.

JACOBI, P.R. Dilemas socioambientais na gestão metropolitana: do risco à busca da sustentabilidade urbana. Política e Trabalho. Revista de Ciências Sociais, n.25, p.115-134, 2006.

KAMMERBAUER, M., MINNERY, J. Risk communication and risk perception: Lessons from the 2011 floods in Brisbane, Australia. Disasters, vol.43, n.1, p.110-134, 2018.

https://doi.org/10.1111/disa.12311

KATES, R.H. Risk assessment of environmental hazard. Chichester: John Wiley, 1978.

KIENZLER, S., PECH, I., KREIBICH, H., MÜLLER, M., THIEKEN, A. H. After the extreme flood in 2002: changes in preparedness, response and recovery of flood-affected residents in Germany between 2005 and 2011, Natural Hazards and Earth System Sciences, vol.15, p.505- 526, 2015. https://doi.org/10.5194/nhess-15-505-2015

KJELLGREN, S. Exploring local risk managers' use of flood hazard maps for risk communication purposes in Baden-Württemberg, Natural Hazards and Earth System Sciences, vol.13, p.18571872, 2013. https://doi.org/10.5194/nhess-13-1857-2013

KUNDZEWICZ, Z. W., KANAE, S., SENEVIRATNE, S. I., HANDMER, J., NICHOLLS, N., PEDUZZI, P., MECHLER, R., BOUWER, L. M., ARNELL, N., MACH, K., MUIR-WOOD, R., BRAKENRIDGE, G. R., KRON, W., BENITO, G., HONDA, Y., TAKAHASHI, K., SHERSTYUKOV, B.: Flood risk and climate change: global and regional perspectives, Hydrological Science Journal, vol.59, p.1-28, 2014. https://doi.org/10.1080/02626667.2013.857411

LAKATOS, E.M., MARCONI, M.A. Fundamentos de metodologia científica. 5. ed. São Paulo: Atlas, 2003.

LAMOND, J., ADEKOLA, O., ADELEKAN, I., EZE, B., UJOH, F. Information for adaptation and response to flooding, multi-stakeholder perspectives in Nigeria. Climate. vol.7, n.4, p.1-18, 2019. https://doi.org/10.3390/cli7040046

LINDELL, M.K., PERRY, R.W. The protective action decision model: Theoretical modifications and additional evidence. Risk Analysis, vol.32, n.4, p.616-632, 2012. https://doi.org/10.1111/j.1539$\underline{6924.2011 .01647 . x}$

LINDSAY, B. R. Social media and disasters: Current uses, future options, and policy considerations. CRS Report for Congress. Washington, DC: Congressional Research Service, 2011.

LUNDGREN, R., MCMAKIN, A. Principles of risk communication. Risk communication: A handbook for communicating environmental, safety, and health risks. Wiley-IEEE Press, p.71-82, 2013. https://doi.org/10.1002/9781118645734.ch6

MAIDL, E., BUCHECKER, M. "Raising Risk Preparedness by Flood Risk Communication." Natural Hazards Earth Systems Science. Vol.15, n.7, p.1577-1595, 2015. https://doi.org/10.5194/nhess-15$\underline{1577-2015}$ 
MANZO, A. J. Manual para la preparación de monografías: una guía para presentear informes y tesis. Buenos Aires: Humanistas, 1971.

MILETI, D.S., SORENSEN, J.H. Communication of Emergency Public Warnings: A Social Science Perspective and State-of-the-Art Assessment. Colorado State University for the Federal Emergency Management Agency, Washington, DC, 1990. https://doi.org/10.2172/6137387

MILETI, D.S., P.W. O'BRIEN. 'Warnings during disaster: normalizing communicated risk'. Social Problems. Vol.39, n.1, p.40-57, 1992. https://doi.org/10.1525/sp.1992.39.1.03×0062j

NEUWIRTH, K., DUNWOODY, S., GRIFFIN, R.J. Protection motivation and risk communication. Risk Analysis, vol.20, n.5, p.721-734, 2000. https://doi.org/10.1111/0272-4332.205065

O'SULLIVAN, J. J., BRADFORD, R. A., BONAIUTO, M., DE DOMINICIS, S., ROTKO, P., AALTONEN, $J$. Enhancing flood resilience through improved risk communications. Natural Hazards and Earth System Sciences, vol.12, p.2271-2282, 2012. https://doi.org/10.5194/nhess-12-2271-2012

PARKER, D.J., TUNSTALL, S.M., MCCARTHY, S. New insights into the benefits of flood warnings: Results from a household survey in England and Wales. Environmental Hazards, vol.7, n.3, p.193210, 2007. https://doi.org/10.1016/i.envhaz.2007.08.005

PARKER, D., PRIEST, S., MCCARTHY, S. Explore surface water flood warning requirements and potential in England and Wales, Applied Geography, vol.31, p.891-900, 2011. https://doi.org/10.1016/i.apgeog.2011.01.002

PING, N.S., WEHN, U., ZEVENBERGEN, C., VAN DER ZAAG, P. Towards two-way flood risk communication: current practice in a community in the UK, J. Water Climate Change, vol.7, n.4, p.651-664, 2016. https://doi.org/10.2166/wcc.2016.015

QUARANTELLI, E. L. Conventional Beliefs and Counterintuitive Realities. Social Research, 75, 873904, 2008

RAAIJMAKERS, R., KRYWKOW, J., VAN DER VEEN, A. Flood risk perceptions and spatial multicriteria analysis: an exploratory research for hazard mitigation. Natural Hazards, vol.46, p.307-322, 2008. https://doi.org/10.1007/s11069-007-9189-z

RENN, O. Risk Governance. Coping with Uncertainty in a Complex World. London: Earthscan, 2008.

ROLLASON, E., BRACKEN, L. J., HARDY, R. J., e LARGE, A. R. G. Rethinking flood risk communication. Natural Hazards, vol.92, p.1665-1686, 2018. https://doi.org/10.1007/s11069-018$\underline{3273-4}$

ROTTENSTREICH, Y., HSEE, C. K. Money, kisses, and electric shocks: An affective psychology of risk. Psychological Science, vol. 12, p.185-190, 2001. https://doi.org/10.1111/1467-9280.00334

SEEBAUER, S., BABCICKY, P. The two faces of social capital in private flood mitigation: Opposing effects on risk perception, self-efficacy and coping capacity. Journal of Risk Research, vol.20, n.8, p.1017-1037, 2017. https://doi.org/10.1080/13669877.2016.1147489

SORENSEN, J.H. Hazard warning systems: Review of 20 years of progress. Natural Hazards Review, vol.1, n.2, p.119-125, 2000. https://doi.org/10.1061/(ASCE)1527-6988(2000)1:2(119)

SORIANO, E., HOFFMANN, W.A.M. A informação e o conhecimento no contexto da comunicação de risco de desastres naturais. Caderno Prudentino de Geografia, Presidente Prudente, v.1, n.37, p.110-123, 2015.

SOUZA, L.B., ZANELA, M.E. Percepção de riscos ambientais: teoria e aplicações. Fortaleza: Edições UFC, 2010. 
STRATHIE, A., NETTO, G., WALKER, G. H., e PENDER, G. How presentation format affects the interpretation of probabilistic flood risk information. Journal of Flood Risk Management, vol.10, n.1, p.87-96, 2017. https://doi.org/10.1111//fr3.12152

TAKEUCHI, Y. Residents Recognition about Rainfall and Evacuation Action in Flood Disaster - Case of Niigata, Fukui Heavy Rainfall and Typhoon No. 23 in 2004, Natural Disaster Research Report, National Research Institute for Earth Science and Disaster Prevention, vol. 40, p. 103-110, 2006

TERPSTRA, T. Emotions, trust and perceived risk: affective and cognitive routes to flood preparedness behavior. Risk Analysis, vol.31, p.1658-1675, 2011. https://doi.org/10.1111/j.1539$\underline{6924.2011 .01616 . x}$

THIEKEN, A. H., KIENZLER, S., KREIBICH, H., KUHLICKE, C., KUNZ, M., MÜHR, B., MÜLLER, M., OTTO, A., PETROW, T., PISI, S., e SCHRÖTER, K. Review of the flood risk management system in Germany after the major flood in 2013. Ecology and Society, vol.21, n.51, p.1-12, 2016. https://doi.org/10.5751/ES-08547-210251

VESTENA, L., GEFFER, E., ALMEIDA, D.E.F., VESTENA, C.L.B. Percepção Ambiental sobre as causas das inundações, Guarapuava/PR: em busca da cidade resiliente. Revista do Departamento de Geografia - USP, vol. 28, p.280-294, 2014. https://doi.org/10.11606/rdg.v28i0.573

VITOUSEK, S., BARNARD, P. L., FLETCHER, C. H., FRAZER, N., ERIKSON, L., STORLAZZI, C. D. Doubling of coastal flooding frequency within decades due to sea-level rise. Nature Scientific Reports, vol.7, p.1399, 2017. https://doi.org/10.1038/s41598-017-01362-7

WHYTE, A.V.T. Perception. In: KA TES, R. W.; AUSUBEL, J. H.; BERBERIAN, M. (Ed.). Climate impact assessment: studies of the interaction of climate and society. Chichester: John Wiley, 1985. p. $107-131$

WINSEMIUS, H. C., AERTS, J. C. J. H., VAN BEEK, L. P. H., BIERKENS, M. F. P., BOUWMAN, A., JONGMAN, B., KWADIJK, J. C. J., LIGTVOET, W., LUCAS, P. L., VAN VUUREN, D. P., WARD, P. J. Global drivers of future river flood risk, Nature Climate Change, vol.6, p.381-385, 2016. https://doi.org/10.1038/nclimate2893

WOODS, M.M., MILETI, D.S., KANO, M., KELLEY, M.M., REGAN, R. AND BOURQUES, L.B. Communicating actionable risk for terrorism and other hazards. Risk Analysis, vol.32, n.4, p.601-615, 2012. https://doi.org/10.1111/j.1539-6924.2011.01645.x

XAVIER, H. Percepção geográfica dos deslizamentos de encostas em áreas de risco no município de Belo Horizonte, MG. 222 f. Tese (Doutorado em Geografia) - Instituto de geociências e Ciências Exatas, UNESP, Rio Claro, 1996.

ZAHARIA, L., IOANA-TOROIMAC, G. Developing soft measures for flood risk mitigation and adaptation in Romania: public informing and awareness. Riscuri si catástrofe. vol.18, n.1, p.7-22, 2016.

Recebido em: 20/05/2020

Aceito para publicação em: 28/10/2020 